This article may be downloaded for personal use only. Any other use requires prior permission of the author and AIP Publishing.

The following article appeared in: Anderson, J.E., Allen, T.M., Garrett, A.W., Gill, C.G., Hemberger, P.H., Kelly, P.B., \& Nogar, N.S. (1997). Resonant laser ablation: Mechanisms and applications. AIP Conference Proceedings, 388, 195-198, and may be found at http://dx.doi.org/10.1063/1.52183. 


\title{
Resonant Laser Ablation: Mechanisms and Applications
}

\author{
J. E. Anderson, T. M. Allen $\dagger$, A. W. Garrett, C. G. Gill, P. H. Hemberger \\ P. B. Kelly ${ }^{\dagger}$ and N. S. Nogar \\ Chemical Sciences and Technology, MS J565, LANL, Los Alamos, \\ New Mexico 87545 \\ (505) 665-7279, FAX(505)665-4631,nogar@lanl.gov
}

tDepartment of Chemistry, University of California, Davis, California 95616

Abstract. We will report on aspects of resonant laser ablation (RLA) behavior for a number of sample types: metals, alloys, thin films, zeolites and soil. The versatility of RLA is demonstrated, with results on a variety of samples and in several mass spectrometers. In addition, the application to depth profiling of thin films is described; absolute removal rates and detection limits are also displayed. A discussion of possible mechanisms for low-power ablation are presented.

\section{Introduction}

Ever since the first report of laser action, it has been recognized that laser ablation (evaporation/volatilization) may provide a useful sampling mechanism for chemical analysis. In particular, laser ablation is rapidly gaining popularity as a method of sample introduction for mass spectrometry. While most laser ablation/mass spectrometry has been performed with fixed frequency lasers operating at relatively high intensities/fluences $\left(\geq 10^{8}\right.$ $W / \mathrm{cm}^{2}, \geq 1 \mathrm{~J} / \mathrm{cm}^{2}$ ), there has been some recent interest in the use of low-power tunable lasers to ablate and resonantly ionize selected components in the ablation plume. This process has been termed resonant laser ablation (RLA) ${ }^{1}$. Potential advantages of RLA include: 1) simplification of the mass spectrum; 2) improvement of the absolute detection limits; and 3) improvement in relative sensitivity.

This work reports on aspects of RLA behavior for a number of sample types, including metals ${ }^{2}$, alloys ${ }^{3}$, thin films ${ }^{4}$, zeolites ${ }^{5}$ and soil. The versatility of RLA for analytical measurements is outlined, with results on a variety of samples and in several mass spectrometers. In addition, the application to depth profiling of thin films is described; absolute removal rates and detection limits are also displayed. An analysis of a variety of fundamental diagnostic experiments and calculations is given, and possible mechanisms for low-power ablation will also be presented.

\section{Experimental}

The experiments presented by this work were conducted using either a quadrupole ion trap mass spectrometer (ITMS) or in a linear and/or reflectron Time of Flight (ToF) mass spectrometer. Both experimental arrangements are based upon excimer pumped dye lasers, using a Questek XeCl excimer : Lumonics HD300 dye laser for the ITMS work and a Lumonics XeCl excimer: Lambda Physik FL2002 dye laser for the ToF experiments. The details of the experimental apparatus may be found elsewhere 2,3 . Figure 1 shows a diagram of the experimental apparatus used for each mass spectrometer. 


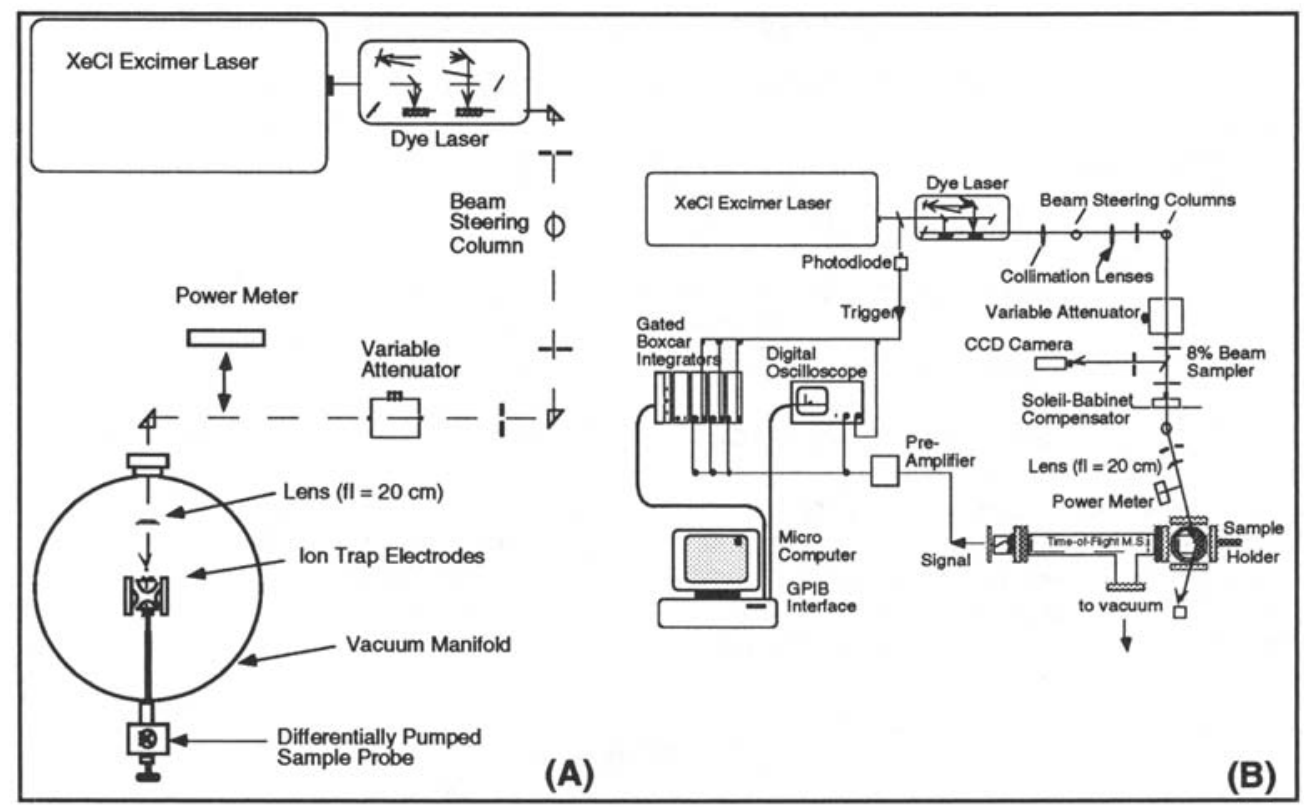

FIGURE 1. Schematic of the ITMS (A) and ToF (B) experimental system.

Samples were prepared as probes for these experiment by machining into a probe tip for metal samples or by bonding thin film wafers to suitable probes with epoxy resin. The powdered samples such as soils or zeolites were bonded to either Si wafers on stainless steel (SS) probes or simply onto the SS probes using a cyanoacrylate ("super glue") based cement.

\section{Mechanistic Studies and Measurements}

The use of relatively low fluence laser pulses $\left(\leq 10^{7} \mathrm{~W} / \mathrm{cm}^{2}\right)$ to sample and selectively ionize analytes from a bulk solid sample has been referred to as resonant laser ablation (RLA) by this and other 6,7 research groups. This method has received considerable attention by this group since this laser based method requires minimal sample preparation (i.e.. no chemical treatments or digests) and affords good discrimination of analyte signal above the background. In RLA, analyte is both desorbed and resonantly ionized by photons from the same laser pulse, with excellent spatial and temporal overlap. Assuming a nominal velocity of $1 \times 10^{5} \mathrm{~cm} / \mathrm{s}^{2}$ and a $15 \mathrm{nsec}$ pulse duration, the spatial extent of the laser generated plume at the sample surface is $\approx 15 \mu \mathrm{m}$, corresponding to a plume density of $\approx 0.1$ Torr. The typical ionization fraction for the " $2+1$ " ionization step is $\approx 10^{-3}$, and the typical desorption yield $10^{-6}$ for the RLA process.

Resonance scans of the various RLA ionization schemes show that the transitions observed are laser linewidth limited at $\sim 0.17 \mathrm{~cm}^{-13}$ but can be readily saturated by small increases in laser power since RLA signals have been observed ${ }^{1}$ to have a slope $\approx 5$ dependence upon laser intensity. By scanning the dye laser across entire transition multiplets for thermometric elements present in a sample and recording spectra, the electronic temperature for RLA can be calculated based upon intensities. For the two photon spectrum of the $a^{5} \mathrm{~F}-\mathrm{e}^{5} \mathrm{~F}$ and $\mathrm{a}^{5} \mathrm{D}-\mathrm{e}^{5} \mathrm{D}$ multiplets of $\mathrm{Fe}(\mathrm{I})$, the calculated electronic temperature for RLA was found to be $\approx 1100 \mathrm{~K}$. 
In addition to the electronic temperature measurements, kinetic energy analysis of the neutrals leaving the sample surface was measured by using a probe laser focused at one of several fixed distances from the sample. By varying the delay between the firing of the ablation laser and the probe laser, velocity distributions were measured. These were then converted to translational temperatures of $\approx 2700 \mathrm{~K}^{2}$. This and the previous measurements do not compare well with the calculated surface temperature of $\approx 400 \mathrm{~K}$ based upon a one dimensional T-Jump calculation; because the observed desorption yield is higher than anticipated for a strictly thermal desorption process, a non-thermal desorption process is suspected.

The relative signal strength observed for RLA is strongly dependent upon the polarization of the laser light, and also upon the surface quality of the samples. For rough sample surfaces (prepared by 500 grit abrasive paper), the signal intensities from RLA reflect the relative intensity of the residual laser light reflected by the sample (S-polarization gave the strongest reflection and ion signal). In contrast, for highly polished surfaces (100 $\AA \mathrm{Cu}$ on Si or diamond polished Cu metal), the intensity of the RLA signal was opposite to that observed for light reflected by the sample. This suggested that although the same trend was observed for reflected light as a function of polarization, the different sample surfaces yielded dramatic differences in ion yield as a function of both surface preparation and polarization. These data are presented in figure 2.

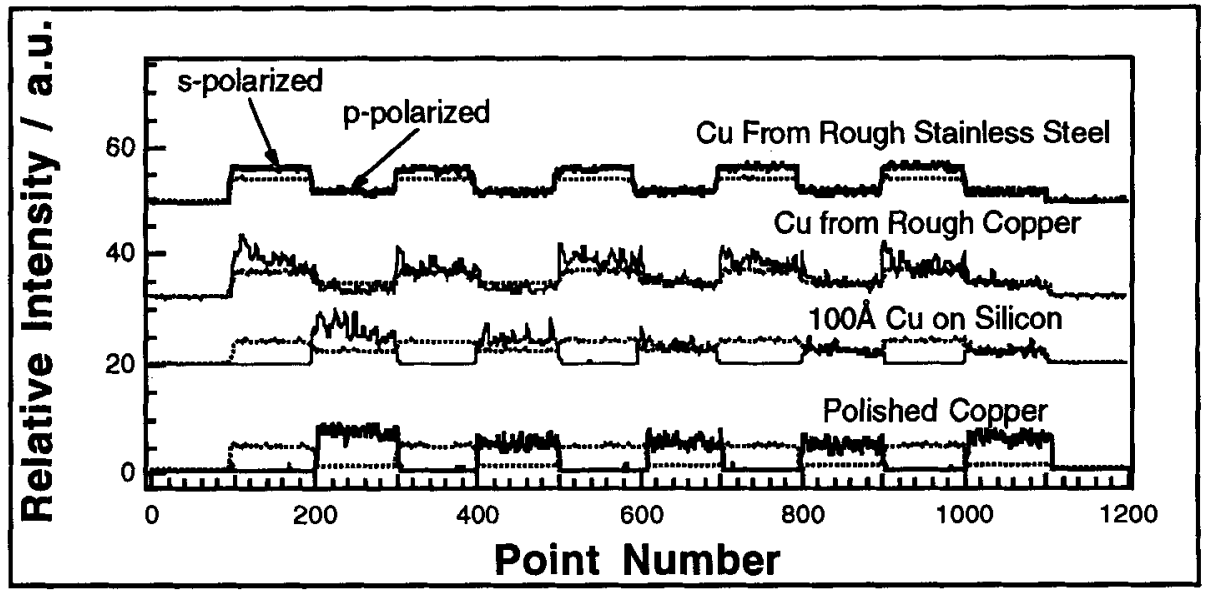

FIGURE 2. Polarization Dependence of RLA signal for different surface qualities. The solid lines represent observed ion signal whereas the dotted lines represent the relative intensity of the laser pulse reflected by the sample surface.

Summarizing the data presented, the material removed from solid samples by the low fluence laser pulses used is much higher than would be anticipated for a strictly thermal process. The wide variation in temperature values determined experimentally from those calculated based upon a thermal desorption mechanism add credibility to a non-thermal desorption hypothesis, and the non-linear intensity dependence of the signal adds further support. The polarization dependence based upon surface preparation suggests that there may be some direct photo physical interaction occurring, such as could be mediated by a surface plasmon interaction. Although surface plasmon interactions are strictly forbidden at planar surfaces, on a microscopic level, the polished surfaces and deposited films have irregularities such as aggregations, kinks and scratches that could act to couple electric field strength from the incident radiation to allow surface plasmon phenomena to occur. 


\section{Analytical Applications of Resonant Laser Ablation}

A number of analytical applications have been addressed by the application of RLA to chemical analysis. By measuring the time (or number of laser shots) required to penetrate copper films of known thickness 2,3 , low fluence removal rates were determined. For typical pulse energies, the average volume removed was $3 \times 10^{-14} \mathrm{~cm}^{3}$ per pulse. Assuming the same density as for bulk copper, this yields an average of $<10^{9}$ atoms/shot, or a depth removal rate of $\approx 10^{-2}$ monolayer/shot and mass removal rate $(M M R)$ of $\approx 10^{-}$ ${ }^{13} \mathrm{~g} / \mathrm{shot}$. Assuming the MMR is similar for copper films as it is for a bulk copper sample, then the absolute detection limits for trace components in copper is given by the product of MMR and the component's concentration. Lead was easily ionized and detected in a NIST (\#494) copper sample at $25 \mathrm{ppm}$ levels, with a $\mathrm{S} / \mathrm{N} \geq 100$, resulting in conservative absolute detection limits in the low attogram range.

The analysis of complex samples by RLA allows the analyst to discriminate analyte signal from those of the bulk matrix, simply by selectively ionizing species of interest. The analysis of trace lead in soil samples was investigated for a NIST (\#4355) Peruvian soil, and for lead concentrations of $129 \mathrm{ppm}$, lead signals were easily observed with high selectivity. This success lead to further investigations in which a series of native soils (Jemez Mts., NM) were spiked with various concentrations of lead. The result of this study was a linear calibration plot for lead over 5 decades of concentration (10 ppb $1000 \mathrm{ppm}$ ), demonstrating the potential for RLA signal quantification.

The analysis of semivolatile (SV) organic compounds is often difficult by mass spectrometry, since these compounds are not readily introduced by either gaseous or direct insertion methods. The use of a selective sorbent material such as a zeolite provides a possible sample handling strategy for laser mass spectrometry on a solid sample support.

Because RLA was successful in selectively generating metal ions for gas phase reactions ${ }^{8}$, metal (silver) ion exchanged mordenite (a zeolite) was used as a selective sorbent for one of the SV compounds of interest to this group, tri-butyl phosphate (TBP). By implementing RLA tuned to a " $2+1$ " transition for silver at $469.84 \mathrm{~nm}$, silver ions were efficiently made, and the reaction of these silver ions with the TBP desorbed by the same laser pulse gave a strong analytical signal for $\mathrm{Ag}(\mathrm{TBP})$ ions $^{5}$, the first mass spectral analysis of TBP that yielded a predominant parent species in the mass spectrum.

Acknowledgements. The authors would like to thank Tom Taylor , Bill Earl and Greg Eiden for helpful discussions and investigations that contributed to further understanding of the mechanistic and/or analytical material presented by this work.

\section{REFERENCES}

1. G. C. Eiden, J. E. Anderson, and N. S. Nogar, Microchem. J. 50, 289-300 (1994).

2. C. G. Gill, T. M. Allen, J. E. Anderson et al., Appl. Opt. 35, 2069-85 (1996).

3. C. G. Gill, A. W. Garrett, P. H. Hemberger et al., Spectrochim. Acta., Part B in press (1996).

4. T. M. Allen, P. B. Kelly, J. E. Anderson et al., Appl. Phys. A 61, 221-225 (1995).

5. C. G. Gill, A. W. Garrett, W. L. Earl et al., JASMS submitted (1996).

6. G. Krier, F. Verdun, and J. F. Muller, Fresenius' Z. Anal. Chem. 322 (4), 379-82 (1985).

7. C. J. McLean, J. H. Marsh, A. P. Land et al., Int. J. Mass Spectrom. Ion Process 96 (1), R1-R7 (1990).

8. C. G. Gill, A. W. Garrett, P. H. Hemberger et al., JASMS 7, 664-7 (1996). 